University of Wollongong

Research Online

Faculty of Engineering and Information

Faculty of Engineering and Information

Sciences - Papers: Part B

Sciences

2019

Effect of cumulative reduction on the microstructure and properties of the NM400/Q345R composite plate

Pengtao Liu

Taiyuan University of Technology, Taiyuan University of Science and Technology

Lifeng Ma

Taiyuan University of Science and Technology

Weitao Jia

Taiyuan University of Science and Technology

Zhengyi Jiang

University of Wollongong, jiang@uow.edu.au

Tao Wang

Taiyuan University of Technology

See next page for additional authors

Follow this and additional works at: https://ro.uow.edu.au/eispapers1

Part of the Engineering Commons, and the Science and Technology Studies Commons

Research Online is the open access institutional repository for the University of Wollongong. For further information contact the UOW Library: research-pubs@uow.edu.au 


\title{
Effect of cumulative reduction on the microstructure and properties of the NM400/Q345R composite plate
}

\author{
Abstract \\ Interfaces and mechanical properties of the NM400/Q345 composite plates fabricated under different \\ reduction ratios were investigated. An increasing in the cumulative reduction ratio enhanced the bonding \\ interface of the NM400/Q345 composite plate by reducing the number of combinations with interfacial \\ defects such as pores. The oxidation film at the interface was crushed and diffused after three passes of \\ rolling (cumulative reduction ratio $=70 \%$ ). The strength of the composite also increased with the \\ increasing of cumulative reduction ratio. At the maximum cumulative reduction ratio ( $70 \%)$, the inter- \\ diffusion of the matrix and composite metal was enhanced, and the microstructure became considerably \\ refined. Furthermore, the elongation of the composite plate was decreased, which can be attributed to the \\ reduced work hardening of the composite plate.

\section{Disciplines} \\ Engineering | Science and Technology Studies

\section{Publication Details} \\ Liu, P., Ma, L., Jia, W., Jiang, Z., Wang, T. \& Zhao, G. (2019). Effect of cumulative reduction on the \\ microstructure and properties of the NM400/Q345R composite plate. Materials Research Express, 6 (9), \\ 096534-1-096534-9.
}

\section{Authors}

Pengtao Liu, Lifeng Ma, Weitao Jia, Zhengyi Jiang, Tao Wang, and Guanghui Zhao 
Effect of cumulative reduction on the microstructure and properties of the NM400/Q345R composite plate Pengtao Liu ${ }^{1,2}$, Lifeng $\mathrm{Ma}^{2,3, *}$, Weitao $\mathrm{Jia}^{2,3}$, Zhengyi Jiang ${ }^{4, *}$, Tao Wang ${ }^{1}$, Guanghui $\mathrm{Zhao}^{2,3}$

${ }^{1}$ College of Mechanical Vehicle Engineering, Taiyuan University of Technology, Taiyuan 030024, Shanxi, China

${ }^{2}$ Heavy Machinery Engineering Research Center of the Ministry Education, Taiyuan University of Science and Technology, Taiyuan 030024, Shanxi, China

${ }^{3}$ College of Mechanical Engineering, Taiyuan University of Science and Technology, Taiyuan 030024, Shanxi, China

${ }^{4}$ School of Mechanical, Materials \& Mechatronic Engineering, NSW 2522, University of Wollongong

Abstract: Interfaces and mechanical properties of the NM400/Q345 composite plates fabricated under different reduction ratios were investigated. An increasing in the cumulative reduction ratio enhanced the bonding interface of the NM400/Q345 composite plate by reducing the number of combinations with interfacial defects such as pores. The oxidation film at the interface was crushed and diffused after three passes of rolling (cumulative reduction ratio $=70 \%$ ). The strength of the composite also increased with the increasing of cumulative reduction ratio. At the maximum cumulative reduction ratio $(70 \%)$, the inter-diffusion of the matrix and composite metal was enhanced, and the microstructure became considerably refined. Furthermore, the elongation of the composite plate was decreased, which can be attributed to the reduced work hardening of the composite plate.

Keywords: Wear-resistant Alloy, Bonding Interface, Mechanical Properties, Composite Plate

*Corresponding authors.

E-mail address: malifengfqh@163.com (Lifeng Ma); jwt860520@163.com (Weitao Jia); jiang@uow.edu.au (Zhengyi Jiang)

\section{Introduction}

Improving the anti-wear ability of materials and the utilization rate of the wear-resistant alloy materials is the major priority in various industrial fields [1]. Friction and wear are commonly observed in coal mining and transportation 
equipment such as integrated coal mining machines, scraper conveyors, and mine hoists. Apart from diverting much manpower and material resources into maintenance and part replacement, the rapid wear of main components poses a serious safety hazard [2].

To mitigate the failure of wear, researchers have investigated the wear performance of various new wear-resistant devices [3] and materials [4]. Valtonen et al. [5] studied the wear behavior of the cutting edge of a mining load-haul-dumper bucket under high-stress abrasion conditions. Das et al. [6] investigated the low-impact abrasion and low-stress sliding wear properties of $\mathrm{Fe}_{17} \mathrm{Mn}$ and $\mathrm{Fe}_{17} \mathrm{Mn}_{3} \mathrm{Al}$. They revealed that hot processing could effectively improve the low-impact wear and low-stress sliding wear of the steel. Ojala et al. [7] reported the wear performance of the quenched wear-resistant steels in abrasive slurry erosion, and Lindroos et al. [8] studied the deformation and wear behavior of four high-strength wear-resistant steels under various impact conditions.

In coal machine equipment, primary wear-resistant parts exhibiting considerable strength, toughness, high-temperature resistance, corrosion resistance, shock resistance, and cost performance can be constructed from the clad metal composites. Therefore, the development of efficient anti-wear composite materials is observed to reduce the wear and tear of coal mine machinery and improve its safety and reliability. Currently, the most common method in industrial production is the rolling composite method [9]. Surface welding technology, which incorporates wear-resistant composite materials into the parts of coal machine equipment, exhibits the disadvantages of poor quality, high cost, and low efficiency [10]. However, the application of clad metal-rolled wear-resistant composite materials is still immature; consequently, the preparation technology of the wear-resistant composite plate materials (which are urgently required for coal machine equipment) is underdeveloped [11]. The high residual stress of the product interface and the low joint strength as well as toughness have hampered the deep processing of such materials.

However, the influence of reduction, heat treatment, and other processes on the bonding surface of the composite plate have not been clearly defined; in particular, 
the composite mechanism of the base metal and wear-resistant steel remains very vague. Zhao et al. [12] reported that the rolling reduction ratio influences the microstructure of the NM360/Q345R composite. Zhi et al. [13] studied the effect of rolling pressure on the microstructure and mechanical properties of the hot-rolled BTW $\backslash$ Q345R composite plates, where the micro-cracks in the interface bonding layer were minimized under a cumulative reduction ratio of $80 \%$. Temperature and strain rate are the main factors that affect the microstructures and mechanical properties of rolled plates [14]. The composite plate cannot always be successfully bitten into the rolls under a large reduction ratio, placing high demands on the equipment and reducing the production efficiency. However, the composite plate cannot always be successfully bitten into the rolls under a large reduction ratio. Otherwise, Better equipment will be required and reducing the production efficiency. Zhi et al. [15] investigated that the loading mode affected the instability mode. To address this deficiency, this paper researches the effect of cumulative reduction on the interface of a rolled composite plate.

Therefore, this study reports the effect of reduction ratio on the bonding interface and mechanical properties of the NM400/Q345R composite plates. After analyzing the micro-hardness, microstructure, and tensile mechanical properties of the composite plates, this study provides technical guidance of the composite interface toughness of the wear-resistant steel composite plates.

\section{Experimental procedures}

In the experiment conducted herein, the composite plate was a rolled plate NM400 $(\mathrm{C} \leq 0.25, \quad \mathrm{Si} \leq 0.7, \mathrm{Mn} \leq 1.6, \mathrm{P} \leq 0.025, \mathrm{~S} \leq 0.01, \quad \mathrm{Cr} \leq 1.4, \quad \mathrm{Mo} \leq 0.5, \mathrm{Ni} \leq 1.0$, $\mathrm{B} \leq 0.004)$ and the substrate was a rolled plate $\mathrm{Q} 345 \mathrm{R}(\mathrm{C} \leq 0.13, \mathrm{Si} \leq 0.44, \mathrm{Mn} \leq 1.52$, $\mathrm{P} \leq 0.018, \mathrm{~S} \leq 0.0012, \mathrm{Al} \leq 0.024)$.

The sheets of steel were cut to a size of $100 \mathrm{~mm} \times 200 \mathrm{~mm} \times 6 \mathrm{~mm}$, and subjected to surface grinding and pickling; then welding assembly. The suction pipe was left in the joint because the gap should be vacuumed before the exhaust pipe was sealed. After vacuuming, the gap between the two surfaces should exist in a vacuum state at a 
pressure of $1 \times 10^{-3} \mathrm{~Pa}$. Images of the fabricated composite plates are denoted in Fig.1.

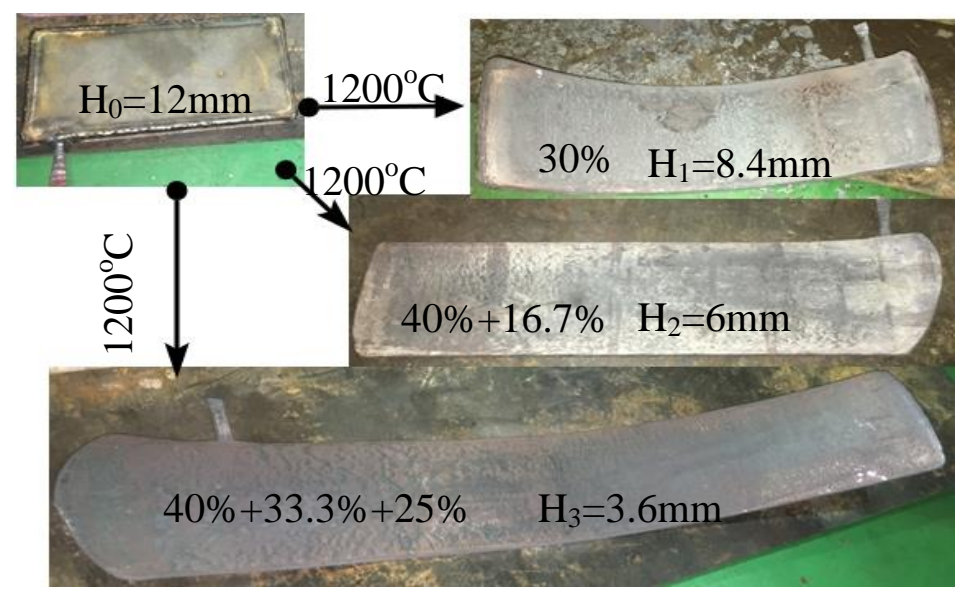

Fig. 1. NM400/Q345 composite plates under different rolling processes

The composite plates were initially placed in a vacuum-atmosphere furnace at $1200^{\circ} \mathrm{C}$ for $20 \mathrm{mins}$, and then rolled and composited via different processes, as depicted in Fig.1. The rolling speed in all the processes was $0.2 \mathrm{~m} / \mathrm{s} .$. The first group of composite plate was rolled once at a reduction ratio of $30 \%$ and a rolling speed of $0.2 \mathrm{~m} / \mathrm{s}$. The second group of composite plate was rolled twice (at reduction ratios of $40 \%$ and $16.67 \%$ in the first and second passes, respectively; cumulative reduction ratio $=50 \%$ ), and the third group of composite plate was rolled three times (at reduction ratios of $40 \%, 33.33 \%$, and $25 \%$ in the first, second, and third passes, respectively; cumulative reduction $=70 \%$ ). The NM400/Q345R composite plates were considered to be the final samples.

The composite plates in each group were inscribed with a metallographic pattern in the thickness direction, ground, and polished. The microstructure was observed by an ultra-depth of field microscope (KeyenceVHX-5000) and a scanning electron microscope (SEM). Tensile samples were obtained from each composite sheet along the rolling direction and subjected to tensile tests on a universal testing machine. The mechanical properties and fracture morphologies of the rolled composite sheets were subsequently derived from the SEM observations of the fractured surfaces.

\section{Influence of the rolling reduction ratio}

\subsection{Analysis of the NM400/Q345R micro-hardness}

Fig.2a denotes the distribution of test points for evaluating the micro-hardness of the NM400/Q345R composite plate. The standard hardness is $\mathrm{HB} \leq 180$ for the carbon 
steel Q345R used herein and $360 \leq \mathrm{HB} \leq 430$ for wear-resistant steel NM400. Fig.2b denotes the hardness value distributions near the bonding interface of the composite plate. The hardness value was significantly higher in the interface when compared with those in the base and cladding layers, and a sharp gradient was observed in case of micro-hardness from the base to the cladding side.

As depicted in Fig.2b, the hardness value was measured across the composite plate perpendicular to the interface. From both the left side (Q345R of the base layer) and the right side (NM400 of the cladding layer), the hardness values gradually increased toward the bonding interface, and were maximized at the interface. The diffusion behavior of the metal elements under high-temperature rolling conditions caused related complex reactions between the constituent elements, forming compounds with large hardness values. The higher hardness of NM400 near the bonding interface when compared with that in the standard NM400 cladding can be attributed to the carbonization effects [16-17]. The hardness of the bonding interface was slightly higher than that of the base layer, indicating that there was a certain degree of work hardening at the interface. The results also verified that the cladding plate has a good combination effect on bonding. As the distances between the interface and the layers on both sides increased, the hardness values tended to be stable and close to the hardness of the layer itself.
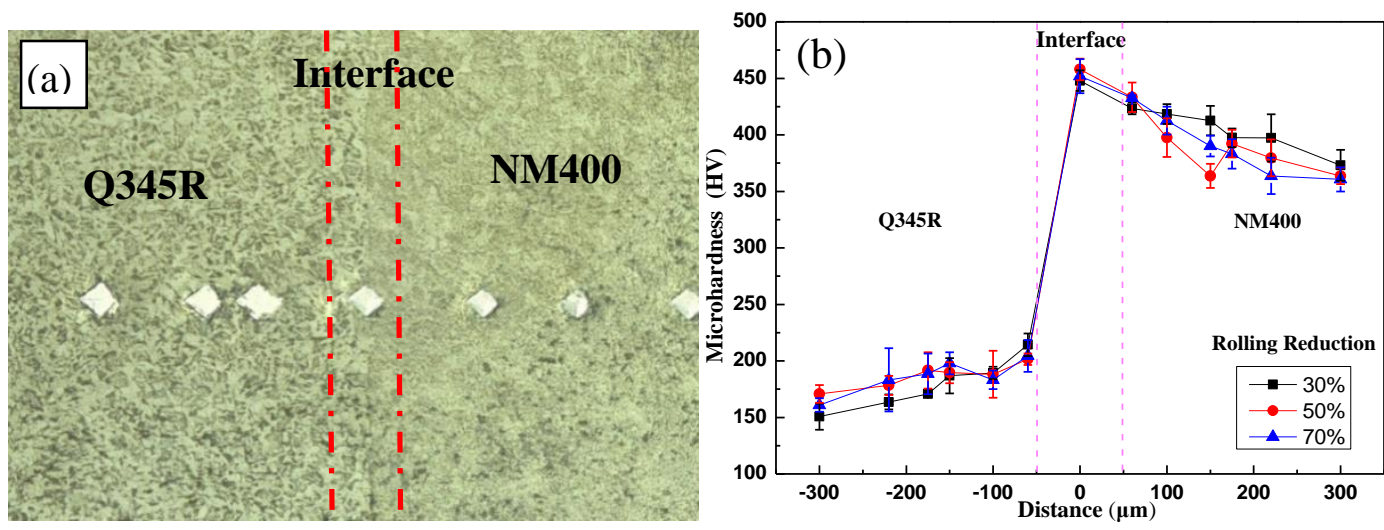

Fig. 2. Hardness distributions of the NM400/Q345R near their interfaces:

(a) distributions of hardness test point; (b) hardness value

The interface hardness (near the NM400 side) increased with the increasing of reduction ratio, which is related with the grain size. The larger the rolling reduction, 
the finer will be the grains and increasing amount of grain boundaries will exist to some extent. The grain boundary hinders the dislocation movement and increases the deformation resistance of the material, increasing the hardness value [18]. The hardness was slightly larger in the composite plate processed at a reduction ratio of $50 \%$ than in the plate processed at a reduction ratio of $70 \%$. This phenomenon is related to the fact that the intermetallic compounds or defects at the bonding interface crushed and dispersed progressively and the hardness of the interface reduced increasing of reduction ratio. The hardness reduction enhanced the bonding strength of the composite interface.

3.2. Effect of rolling reduction ratio on the microstructure
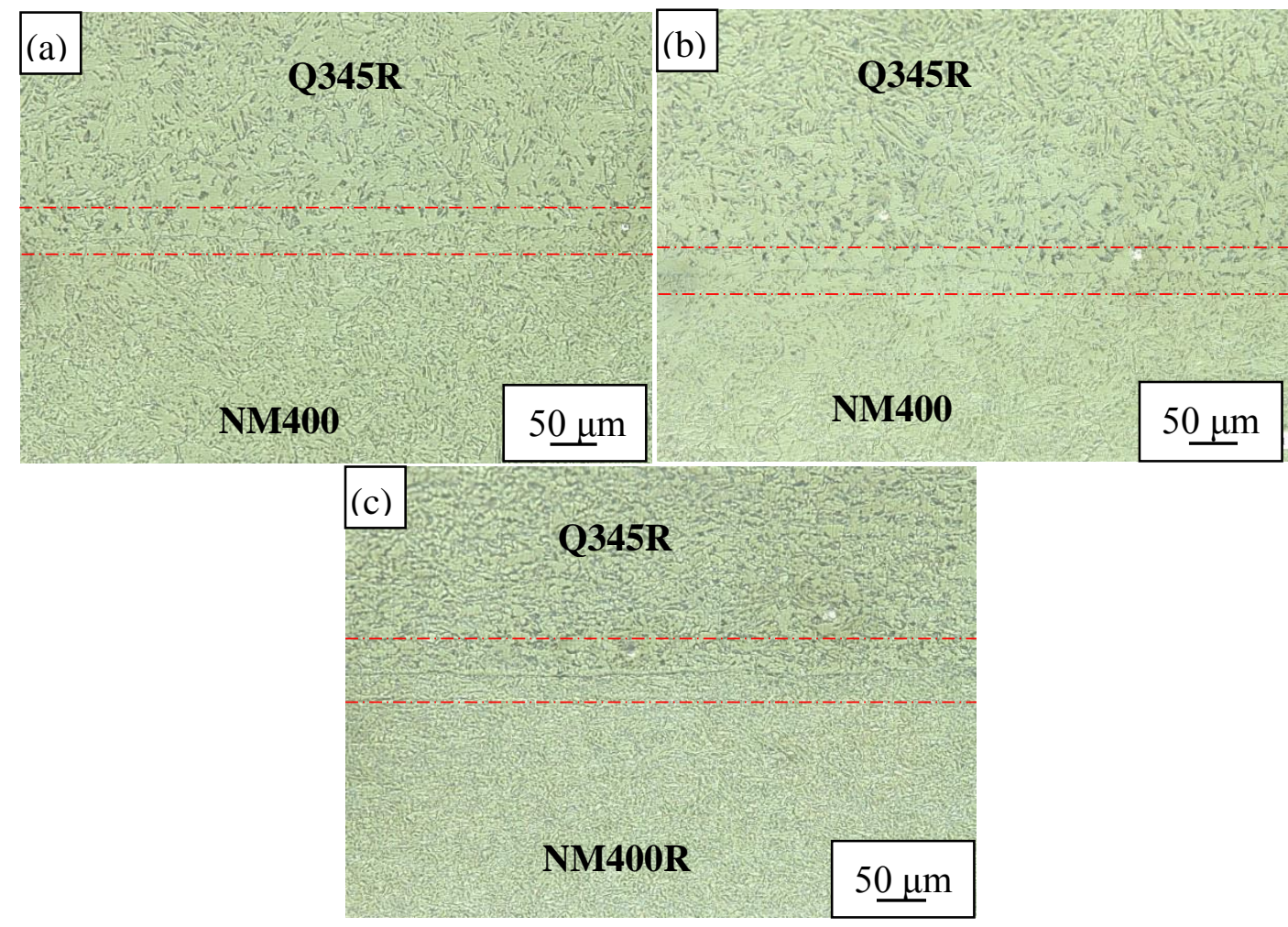

Fig. 3. Microstructures of the NM400/Q345R composites under different cumulative reduction ratios:

(a) $30 \%$; (b) $50 \%$; (c) $70 \%$

Fig.3 denotes the metallographic bonding interfaces of the NM400/Q345R composite plates processed at different reduction ratios. As can be observed from Fig. $3 \mathrm{a}$, the metals of the composite processed at a reduction ratio of $30 \%$ were mainly separated at the interface, and the performance of bonding between the base and 
cladding layers was poor. An increase in the reduction ratio improved the diffusion of the elements together with the bonding force at the interface (Fig.3b and c). Most of the structures on the Q345R side were ferrites and pearlites. As the reduction ratio increased, the grain size near the interface of the composite plate elongated in the rolling direction. The grain size of the base layer Q345R and the cladding layer NM400 continuously decreased as the reduction ratio increased, and the pearlites changed from coarse to fine and uniform. This phenomenon was caused by grain recrystallization under the combined influence of large plastic deformations and the high temperature of rolling, which refined the microstructure and improved the mechanical properties. The results indicated that increasing the cumulative reduction strengthened the bonding of the NM400/Q345R composite and refined the microstructure to a certain extent [19].

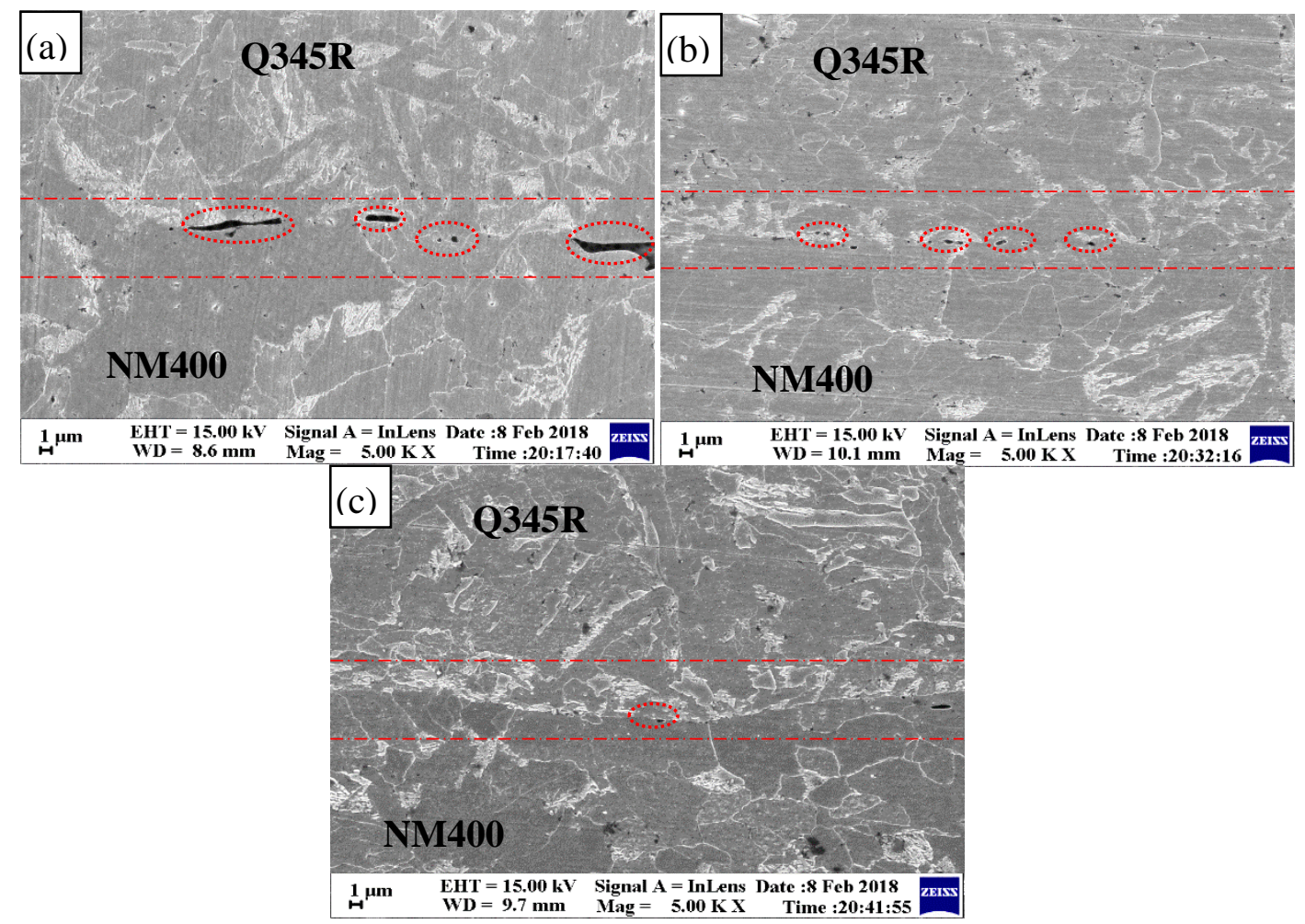

Fig. 4. Scanning electron microscope images of the NM400/Q345R composites under different cumulative reduction ratios: (a) $30 \%$; (b) $50 \%$; and (c) $70 \%$

Interfacial recombination in the wear-resistant steel-composite plate was further examined through SEM observations of the composite interface (see Fig.4). The NM400/Q345R interface, which was processed at a reduction ratio of 30\%, contained defects such as holes and granules (Fig.4a). The granular defects may be due to the 
low reduction ratios, when the interfacial metal flow is poor and the formed compound is incompletely crushed, which reduces the interfacial bonding. In the samples processed at cumulative reduction ratios of 50\% (Fig.4b) and 70\% (Fig.4c), the number of defects at the composite interface reduced; particularly in Fig.4c, the defects dwindled to only few small granular defects, and no clear interface is visible. This indicated a good composite effect, high homogeneity, and refined grains in the composite plate processed at high reduction ratio. Based on the interfacial microstructures of the NM400/Q345R composite plates processed at different rolling reduction ratios, it was concluded that the ferrite and pearlite structures were evenly distributed near the interface, and pearlites were distributed in a typical sheet shape. As the rolling reduction ratio increased, the size of pearlites and ferrites changed from coarse to refined. The structure was identical in the upper and lower part. The complete grain structure could be observed throughout the original interface, indicating the formation of strong metallurgical bonds on the interface [20-21].

\section{Effect of the rolling reduction ratio on the mechanical properties}

\subsection{Analysis of the mechanical properties of NM400/Q345R}

Fig.5a shows that the tensile stress-strain curves of the composite plates processed at different reduction ratios. The tensile strength of the composite plate increased with increasing reduction ratio, but the increase was small (Fig.5b). When the cumulative reduction became $70 \%$, the maximum tensile strength of the sample became 558.01 MPa. Conversely, the elongation decreased with increasing reduction ratio (see Fig.5c) and was minimized at $70 \%$. This trend can be attributed to the increased deformation amount under high reduction ratios. Severe plastic deformation of the composite plate was also accompanied by work hardening, which became increasingly severe at higher cumulative reduction ratios. Consequently, the strength decreased, whereas the elongation increased. On the one hand, the strength of the composite was reduced by work hardening. On the other hand, the grain refinement generated by the rolling force improved the mechanical properties of the material. 

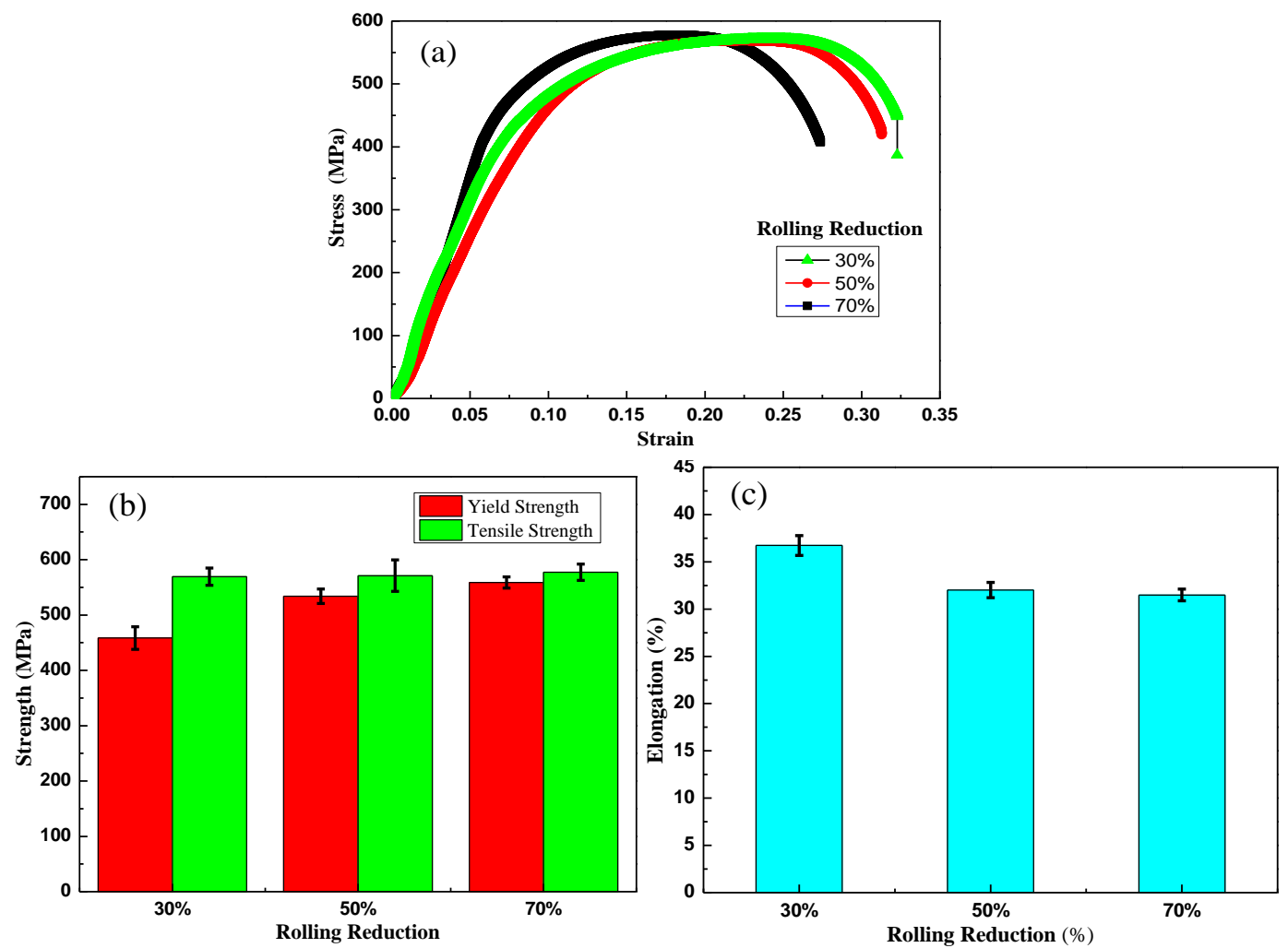

Fig. 5. Mechanical properties of NM400/Q345R under different cumulative reduction ratios:

(a) stress-strain curves; (b) strength results; (c) elongation results

\subsection{Analysis of fracture morphology near the interface}

The SEM images of the tensile fractures in the NM400/Q345R composite under different reduction conditions can be illustrated in Fig.6. The interface was more stratified in the sample processed at a cumulative reduction ratio of $30 \%$ (Fig.6a) when compared with that in the sample processed at a reduction ratio of 50\% (Fig.6b), displaying slight interface stratification. In the sample processed at a reduction ratio of $70 \%$ (Fig.6c), interfacial layered cracks were absent and the tensile fracture characteristics confirmed a strong interface. Multiple microstrip cracks appeared along the rolling direction along with several fine pits. These features were complex fracture modes exhibiting the characteristics of cleavage and ductile fracture. However, as the reduction ratio increased, the pits gradually enlarged and became more even. Thus, a large cumulative reduction ratio can achieve the full diffusion behavior of the interfacial alloy elements. An increase in the cumulative reduction ratio improved the interfacial bonding strength, enhancing the toughening effect of the composite interface. 

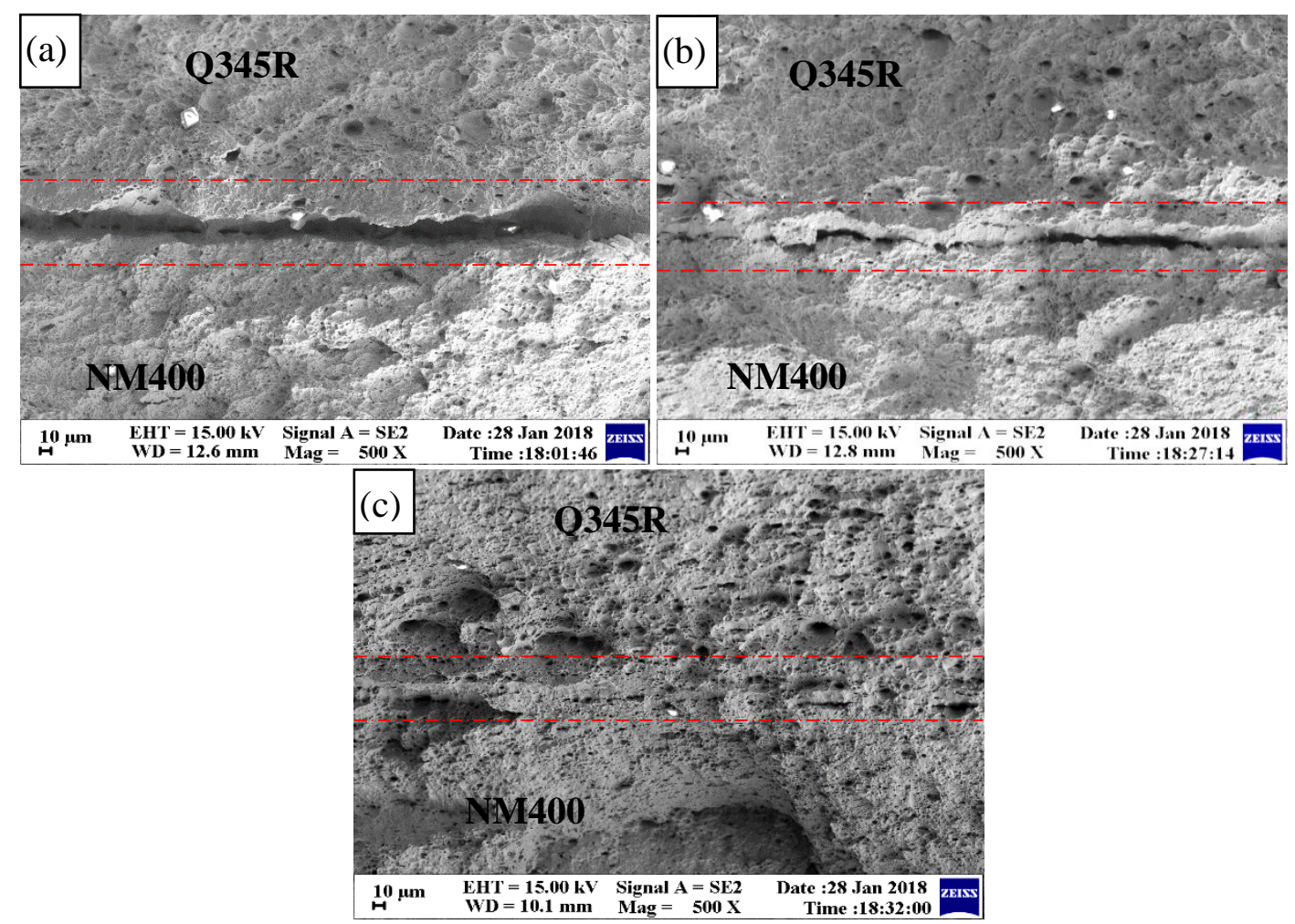

Fig. 6. Fracture morphology near the interface of NM400/Q345R under different rolling reduction ratios:

(a) $30 \%$; (b) $50 \%$; (c) $70 \%$
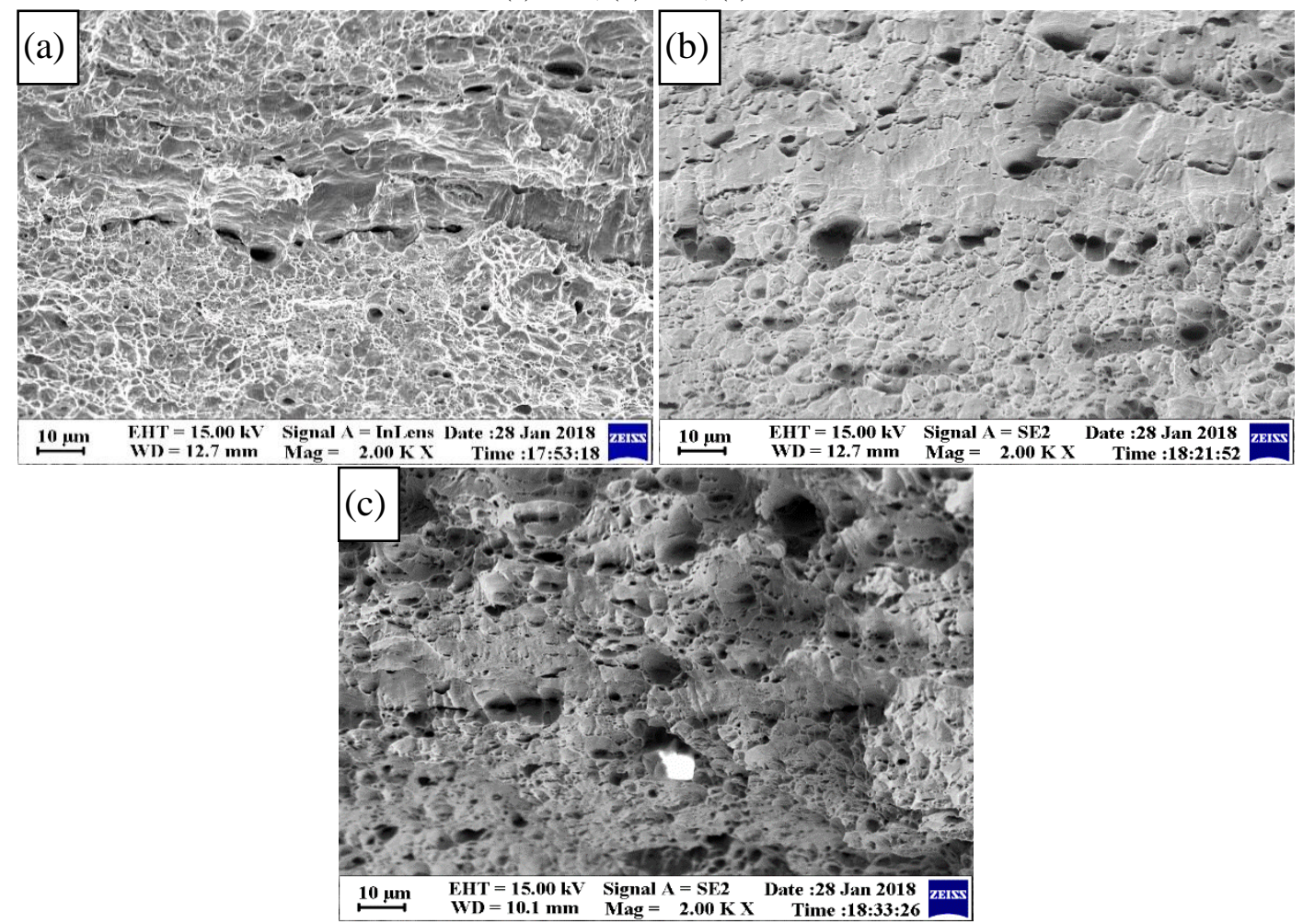

Fig. 7. Fracture morphologies of Q345R under different cumulative reduction ratios:

(a) $30 \%$; (b) $50 \%$; (c) $70 \%$

Fig.7 reveals that the tensile fracture morphologies at the Q345R side of the samples processed at different cumulative reduction ratios. Dimples of different sizes 
and inconsistent depths were distributed around the near-interface fractures in all the samples. Small dimples were observed to gather around larger dimples. Because the inclusions were concentrated by tensile stress, composite plates were prone to fracture. As the reduction ratio increased, the dimple size tended to decrease. The number of small-sized dimples increased, whereas the number of irregularly shaped carbide particles decreased, indicating the gradual disappearance of the stress concentration. Based on the microstructure of the carbon steel side near the interface, increasing the cumulative reduction did not alter the internal composition of the matrix, and the carbide distribution remained relatively uniform. The samples resisted the external stretching force and diffused the stress concentration. Ultimately, the tensile strength of the composite was enhanced [22-23].
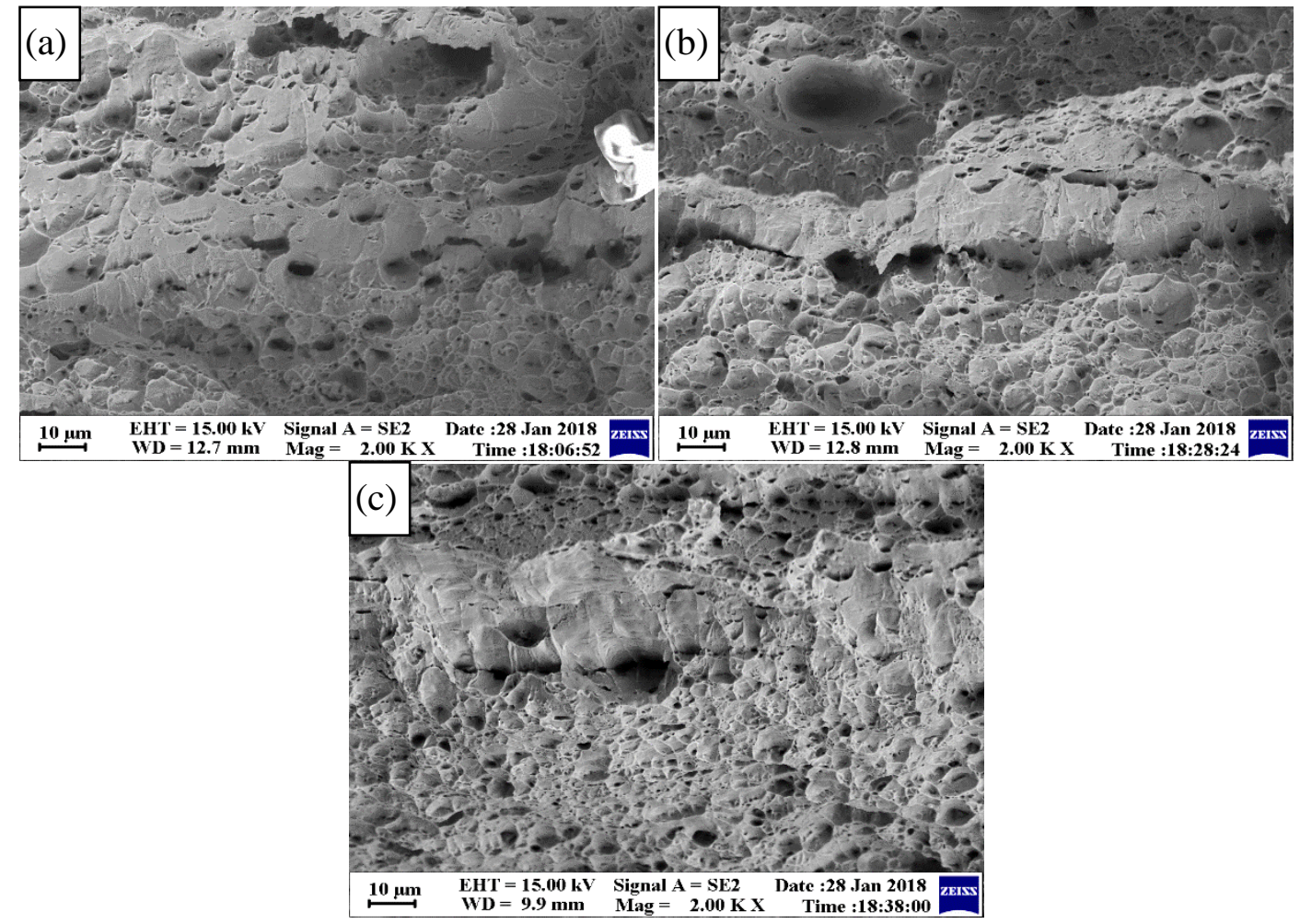

Fig. 8. Fracture morphologies of NM400 under different cumulative reduction ratios: (a) $30 \%$; (b) $50 \%$; (c) $70 \%$

Fig.8 displays the fracture characteristics at the NM400 substrate side of the composite under different cumulative reductions. The surface of the NM400 substrate fracture contained dimples of inconsistent size and shape. The dimples had significant depth and were approximately $2 \mu \mathrm{m}$ in diameter. The fractures contained fewer "river-like patterns" than those in the Q345R steel, indicating fewer brittle fractures 
during the stretching process and the high plasticity of the composite sheet. At a cumulative reduction ratio of $30 \%$, the dimples in the NM400 fractures were coarse and uneven. At a reduction ratio of $70 \%$, the dimples increased in number and reduced in size, indicating that deformation improved the strength of the NM400 substrate.

\section{Conclusions}

(1) The microhardness value was the highest at the interface of the NM400/Q345R composite plate. This was because a high-hardness compound was formed at the interface even though work hardening also occurred therein. As the cumulative reduction ratio increased, the hardness at the interface initially increased and then decreased. Under a cumulative reduction ratio of 30\%, many defects (such as holes) were observed at the interface. Therefore, the hardness value was the smallest at the intermediate reduction ratio $(50 \%)$ when the defects were compacted but not properly crushed. Under a cumulative reduction ratio of $70 \%$, the compounds were thoroughly crushed and diffused, reducing the interface hardness and improving the interface bonding status.

(2) As revealed in the microstructure observations of the NM400/Q345R composite plate, increasing the reduction ratio improved the mutual diffusion of the constituents at the bonding interface, enhancing the composite effect. The NM400 and Q345R constituents can realize a wide range of metallurgical bonding layers. The oxide at the interface gradually disintegrated as the deformation increased, reducing the quantity of oxide along with the grain size. Finally, increasing the reduction ratio promotes the formation of bonding points at the interface, improving the bonding quality of the NM400/Q345R composite.

(3) Increasing the cumulative reduction ratio significantly affected the mechanical properties of the NM400/Q345R composite plates; in particular, the tensile strength of the plates gradually increased and their elongation reduced. The tensile strength of $\mathrm{NM} 400 / \mathrm{Q} 345 \mathrm{R}$ was maximized at $1200^{\circ} \mathrm{C}$ and a cumulative reduction ratio of $70 \%$. In the sample processed under these conditions, no cracking was observed at the bonding interface after stretching, and the interface exhibited a 
strong toughening effect. The rolling composite performance was relatively higher under these conditions when compared with that under the remaining processing conditions.

\section{Acknowledgments}

This work was supported by the Applied Basic Research Project of Shanxi Province (201701D121078 and 201701D221143), Major program of national natural science foundation of China (U1710254), Shanxi province science and technology major projects (MC2016-01), and Doctoral Scientific Research Start-up Foundation of Taiyuan University of Science and Technology of China (20192002).

\section{References}

1. Holmberg K, Kivikyto-Reponen P, HaRkisaari P, et al. Global energy consumption due to friction and wear in the mining industry[J]. Tribology International, 2017(115):116-139.

2. Zambrano O A, Gómez J A, Coronado J J, et al. The sliding wear behaviour of steels with the same hardnes[J]. Wear, 2019(418-419):201-207.

3. Technology S, Anshan, Shun C W Y, et al. A new type of corrosive wear test device and its performance study [J]. Journal of Materials Engineering, 2000(5):27-30.

4. Kumar S, Mondal D P, Jha A K, et al. Improvement in high stress abrasive wear property of steel by hardfacing $[\mathrm{J}]$. Journal of Materials Engineering and Performance, 1999(8):711-715.

5. Valtonen K, Keltamäki K, Kuokkala V T. High-stress abrasion of wear resistant steels in the cutting edges of loader buckets [J]. Tribology International, 2018(119):707-720.

6. Dalai R, Das S, Das K. Effect of thermo-mechanical processing on the low impact abrasion and low stress sliding wear resistance of austenitic high manganese steels [J]. Wear, 2019(420-421):176-183.

7. Ojala N, Valtonen K, Antikainen A, et al. Wear performance of quenched wear resistant steels in abrasive slurry erosion [J]. Wear, 2016(354-355):21-31. 
8. Lindroos M, Ratia V, Apostol M, et al. The effect of impact conditions on the wear and deformation behavior of wear resistant steels [J]. Wear, 2015(328-329):197-205.

9. Eslami P, Karimi Taheri A, Zebardast M, et al. A comparison between cold-welded and diffusion-bonded $\mathrm{Al} / \mathrm{Cu}$ bimetallic rods produced by ECAE process [J]. Journal of Materials Engineering and Performance, 2013(22):3014-3023.

10. Mendez P F, Barnes N, Bell K, et al. Welding processes for wear resistant overlays [J]. Journal of Manufacturing Processes, 2014(16):4-25.

11. Liu S Y, Wang A Q, Lv S J, et al. Interfacial properties and further processing of $\mathrm{Cu} / \mathrm{Al}$ laminated composite: a review [J]. Materials Review, 2018(32):828-835.

12. Zhao G H, Ma L F, Huang Q X, et al. Microstructure evolution and recrystallization analysis of hot rolled NM360/Q345R composites [J]. Materials Research Express, 2018(5).

13. Zhi C C, Ma L F, Huang Q X, et al. Effect of reduction on bonding interface of hot-rolled wear-resistant steel BTW1/Q345R cladding plate [J]. Journal of Wuhan University of Technology (Materials Science), 2018 (33): 952-958.

14. Jia W T, Ma L F, Le Q C, et al. Deformation and fracture behaviors of AZ31B Mg alloy at elevated temperature under uniaxial compression [J]. Journal of Alloys and Compounds, 2019(783):863-876.

15. Zhi C C, Ma L F, Huang Q X, et al. Improvement of magnesium alloy edge cracks by multi-cross rolling $[\mathrm{J}]$. Journal of Materials Processing Technology, 2018(255):33-339.

16. Liu B X, Wang S, Chen C X, et al. Interface characteristics and fracture behavior of hot rolled stainless steel clad plates with different vacuum degrees [J]. Applied Surface Science, 2019(463):121-131.

17. Zhu Z, He Y, Zhang X, et al. Effect of interface oxides on shear properties of hot-rolled stainless steel clad plate [J]. Materials Science and Engineering: A, 2016(669):344-349.

18. Sharma G, Dwivedi D K. Effect of pressure pulsation on bond interface characteristics of 409 ferritic stainless steel diffusion bonds [J]. Vacuum, 
2017(146):152-158.

19. Ding Z Y, Wang X G, Gao Z L, et al. An experimental investigation and prediction of fatigue crack growth under overload/underload in Q345R steel [J]. International Journal of Fatigue, 2017(98):155-156.

20. Jiang J, Ding $\mathrm{H}$, Luo $\mathrm{Z}$ A, et al. Interfacial microstructure and mechanical properties of stainless steel clad plate prepared by vacuum hot rolling [J]. Journal of Iron and Steel Research International, 2018(25):732-738.

21. Murugan S P, Cheepu M, Nam D G, et al. Weldability and fracture behaviour of low carbon steel/aluminium/stainless steel clad sheet with resistance spot Welding [J]. Transactions of the Indian Institute of Metals, 2017(70):759-768.

22. Wei S P, Wang G, Yu J C, et al. Competitive failure analysis on tensile fracture of laser-deposited material for martensitic stainless steel[J]. Materials \& Design, 2017(118):1-10.

23. Dhib Z, Guermazi N, Ktari A, et al. Mechanical bonding properties and interfacial morphologies of A283/316 clad composite [J]. Materials Science \& Engineering A, 2017(696):374-386. 